\title{
ORIGINAL
}

\section{EPIDEMIOLOGÍA DE LA VARICELA EN ESPAÑA EN LOS PERÍODOS PRE Y POST VACUNACIÓN}

\author{
Isabel Peña-Rey (1, 2), María Victoria Martínez de Aragón (1, 2), Ana Villaverde Hueso (3), \\ Monserrat Terres Arellano (1), Enrique Alcalde Cabero (1) y Berta Suárez Rodríguez (1).
}

(1) Centro Nacional de Epidemiología. Instituto de Salud Carlos III.

(2) Ciber de Epidemiología y Salud Pública.

(3) Instituto de Investigación de Enfermedades Raras. Instituto de Salud Carlos III.

\section{RESUMEN}

Fundamento: El virus varicela zoster puede causar dos enfermedades, la varicela y el herpes zóster. La vacuna frente a la varicela se incorporó en España en 2005 para personas susceptibles de entre 10 y 14 años. En 2007 se aprobó una propuesta de vigilancia de la varicela y herpes zóster que permitiera detectar posibles cambios en los patrones de distribución por edad, en la gravedad y complicaciones. El objetivo de este trabajo es conocer la carga de enfermedad por varicela antes y después de la vacunación.

Método: Se analizan los datos agregados (casos e incidencia) de varicela y herpes zóster en España en el sistema CMBD para 1997-2003 y 2005-2007, así como la mortalidad por esta enfermedad a nivel nacional para e período1999-2006.

Resultados: El 88,1\% de los casos de varicela se da en personas menores de 15 años. En el CMBD se registró un promedio anual de 1.311 ingresos. No se observaron cambios significativos en la distribución por edad, sexo ni complicaciones durante los ingresos en ninguno de los períodos estudiados. El $32-36 \%$ anual ingresó por un motivo diferente a varicela. La mortalidad osciló entre 4 y 14 individuos/año, el 80\% mayores de 14 años. El 64\% de los casos notificados de herpes zóster fueron mayores de 50 años. La tasa media anual de ingresos por fue de 2,5 por 100.000 habitantes sin diferencias por sexo. La tasa media anual de defunciones fue 0,31 por millón de habitantes.

Conclusiones: En España la varicela tuvo una disminución generalizada durante 2005-2007, pero es difícil valorar el impacto de la vacunación por la falta de cifras de cobertura vacunal y porque este período coincide con el inmediato a la última onda epidémica, cuyo máximo se registró en 2004.

Palabras clave: Varicela. Herpes zoster. Incidencia. Morbilidad. Mortalidad. impacto vacuna.

Correspondencia:

Isabel Peña-Rey

Centro Nacional de Epidemiología

Instituto de Salud Carlos III

Avda Monforte de Lemos, 5

Tlfo:91 8222636. Fax: 913877815

sabela@isciii.es
ABSTRACT

\section{Epidemiology of Varicella in Spain Pre-and Post-vaccination Periods}

Background. Varicella virus can cause two different diseases: chickenpox and herpes zoster. In 2005 varicella vaccine has been introduced in the Spanish national vaccination schedule for 10-14 years old non-immune people, in order to reduce the severity of the disease. In 2007 a new surveillance protocol with aggregate data for chickenpox and herpes zoster was approved in order to detect any change in age distribution, severity and complications of the chickenpox and herpes zoster cases. The aim of this study is to know the burden of diseases (in the last ten years).

Methods. Number of cases, hospitalization and incidence for chickenpox and herpes zoster were study for two periods 1997-2003 and 2005-2007. Analysis for 1996-2007 fatal cases was done too. We decided to remove year 2004 because the extremely high chickenpox incidence registered. Sources of data: RENAVE (Spanish Surveillance Network), Spanish hospital surveillance system (CMBD), and mortality registries.

Results. Chickenpox incidence decreased since 2005, but an increasing trend was detected in hospitalisation with an average of 1,311 hospitalizations every year. For the 32\%-36\% of hospitalized cases, the main diagnosis was not chickenpox. 4-14 deaths per year have been detected; $80 \%$ of them were older than 14 years. Annual rate of herpes zoster hospitalization was 2.5 per 100,000 inhabitants, similar in both sexes. Case fatality rate per year was 0.31 per million inhabitants. No significant changes were detected in age and sex in complicated cases between the two periods. $88 \%$ of chickenpox cases were younger than 15 years old and $64 \%$ of herpes zoster older than 50 years in 2007.

Conclusions. Chickenpox has been decreasing during 2005-2007 in Spain. The impact of vaccination is difficult to asses, because of a peak registered in 2004 but also because the lack of vaccination coverage information for this period and the case-data information is available only for the last year.

Keywords: Chickenpox. Herpes zoster. Incidence. Morbidity. Mortality. Vaccine impact. 


\section{INTRODUCCIÓN}

La varicela es una enfermedad infectocontagiosa de distribución mundial, producida por la infección primaria del virus varicela zoster (VVZ) o herpesvirus humano 3, y es característica de la edad infantil. El ser humano es el único reservorio conocido. En climas templados más del $90 \%$ de la población la ha padecido antes de los 15 años y un $95 \%$ en el comienzo de la vida adulta. En estos países la varicela presenta un patrón estacional característico con epidemias anuales en invierno y principios de primavera. En climas tropicales el patrón no es tan evidente, el virus circula con menos frecuencia y la enfermedad se adquiere en edades más tardías ${ }^{1-3}$.

En general es una enfermedad leve pero en algunos casos puede complicarse, especialmente en los bebés, en los adultos y en personas inmunodeprimidas ${ }^{4}$. Las complicaciones pueden ser cutáneas (sobreinfecciones bacterianas de las lesiones), del sistema nervioso (encefalitis, meningitis), respiratorias, etc. La neumonía es la complicación más común en los adultos enfermos y requiere hospitalización en uno de cada 400 casos de varicela aproximadamente ${ }^{4}$.

El herpes zoster (HZ) es la manifestación local de la reactivación del VVZ, que después de la primoinfección queda latente en los ganglios de las raíces dorsales de la médula espinal. Se produce cuando hay una disminución de la inmunidad celular, lo que puede ocurrir de forma natural, como resultado de la edad o como consecuencia de tratamientos y/o enfermedades que inducen inmunosupresión. Puede dejar como secuela una neuralgia posherpética (NPH). La infección permanece latente en la mayoría de los primoinfectados por el virus, y entre el 15\%-20\% de ellos desarrolla el HZ en algún momento de su vida ${ }^{5,7-}$ 8 . No presenta variación estacional, siendo una enfermedad de aparición esporádica.

El diagnóstico de ambas enfermedades es clínico y el tratamiento, sintomático, trata de evitar las complicaciones. En el caso del HZ el tratamiento precoz con antivirales disminuye la severidad y la duración del dolor agudo así como la duración de la fase aguda de la NPH, pero no tiene impacto en el riesgo de desarrollar la NPH.

La vacuna antivaricela fue registrada inicialmente para uso exclusivo en pacientes inmunodeprimidos (en Europa en 1984 y en Japón en 1986). Posteriormente se autorizó para uso general en Japón y Corea del Sur en 1988 y en los Estados Unidos de América, Suecia y Alemania en $1995^{8,9}$. Actualmente existen dos presentaciones de la vacuna procedentes de la misma cepa vacunal (OKA). Se han realizado varios estudios pre y postexposición a la vacuna y la mayoría de las estimaciones aseguran que con una dosis la protección es del 85\% (rango 44\%-100\%) para prevenir todo tipo de varicela y del $100 \%$ para prevenir la varicela severa. Cuando la vacuna se administra después de la exposición a la enfermedad se ha demostrado altamente efectiva en la prevención o modificación de la misma ${ }^{10}$. Aunque este nivel de efectividad se considera alto, para conseguir la interrupción de la transmisión de la enfermedad se necesitan dos dosis de vacuna ${ }^{11}$.

Recientemente se ha autorizado la vacuna cuatrivalente frente a sarampión, rubéola parotiditis y varicela. Existen dos presentaciones comerciales: una de uso entre los 12 meses y los 12 años y otra autorizada en niños menores de 2 años. La vacuna se registró en España para uso exclusivo en pacientes de alto riesgo en 1997 y en 2005 se recomendó su inclusión en el calendario vacunal para niños de entre 10-14 años susceptibles, previa detección de anticuerpos negativos y a los contactos próximos sanos de los pacientes susceptibles de riesgo, con el objetivo de disminuir la incidencia de la enfermedad en edades en las que las complicaciones son más graves y hay más mortali$\operatorname{dad}^{12}$. Dos Comunidades Autónomas (CCAA): Madrid en noviembre de 2006 y Navarra en enero de 2007, y Ceuta y Melilla 
en 2008 han incorporado además la vacunación universal a los 15 meses de edad ${ }^{13}$.

En España las encuestas de seroprevalencia realizadas en 1996, tanto a nivel nacional como en algunas CCAA, indican que a los 5 años de edad el $50 \%$ de la población ya ha sido infectado por el virus y se encuentra protegida, porcentaje que aumenta con la edad, llegando al $90 \%$ en el grupo de $10-15$ años $^{14}$.

En España desde 1904 la varicela es una enfermedad de declaración obligatoria (EDO) y desde el año 1997 con la creación de la Red Nacional de Vigilancia Epidemiológica (RENAVE) se notifica el número de casos por semana y año ${ }^{7,12}$. Algunas CCAA realizan además una vigilancia centinela de varicela y $\mathrm{HZ}$ obteniendo datos epidemiológicos básicos de cada enfermo. Con el objetivo de evaluar el impacto de los programas de vacunación establecidos y detectar puntos críticos, la introducción de una vacuna en el calendario vacunal debe ir acompañada de un sistema adecuado de vigilancia que permita conocer la incidencia de la enfermedad y su evolución por edad, así como detectar cambios en los patrones de distribución y en la gravedad de los casos. Por ello, en el año 2007 se estableció un nuevo protocolo de vigilancia a nivel nacional con la notificación de casos agregados por edad y sexo de varicela y HZ, e información del estado vacunal ${ }^{15}$.

El objetivo del estudio es conocer la situación epidemiológica de la varicela y HZ en España en el período prevacunal y a los dos años de la inclusión de la vacuna, para evaluar su impacto.

\section{MATERIAL Y MÉTODO}

Estudio descriptivo retrospectivo de la varicela y $\mathrm{HZ}$ basado en el análisis de la incidencia, la morbilidad y la mortalidad de la enfermedad en España.
Fuentes de datos: Red Nacional de Vigilancia Epidemiológica (RENAVE), Conjunto Mínimo Básico de Datos al Alta Hospitalaria (CMBD), base de datos disponible en el Centro Nacional de Epidemiología (CNE) y Registro de Mortalidad del Instituto Nacional de Estadística (INE). Las poblaciones anuales por CCAA se obtuvieron de las proyecciones censales del INE.

Para el análisis de la incidencia se obtuvo el número de casos totales de varicela de la RENAVE (1997-2007). Hasta el año 2006 la información es numérica, para el año 2007 se dispone de la información desagregada por grupo de edad para varicela, de 9 CCAA siguiendo el nuevo protocolo de vigilancia. Para estimar la incidencia por edad a nivel nacional en los años previos se ha asumido la distribución por edad de los datos disponibles de la vigilancia centinela de algunas CCAA o se ha estimado a partir de los registros informatizados de Atención Primaria.

Para el HZ no se dispone de datos de incidencia a nivel nacional, pero en 2007 se obtuvo información desagregada por edad y sexo de 5 CCAA.

Para conocer la morbilidad de la enfermedad para el período 1997-2007 se utilizó el CMBD, que recoge el diagnóstico al alta basado en la causa que motivó el ingreso, y otros diagnósticos concurrentes en el momento del ingreso o que se desarrollen a lo largo de la estancia hospitalaria. Se ha realizado la búsqueda de las personas ingresadas que presentaran en alguno de los 10 posibles campos diagnósticos algún código de varicela incluidas sus complicaciones $(052,0-052,9)$ y del HZ $(053,0-053,9)$ según la Clasificación Internacional de Enfermedades (CIE-9-MC) ${ }^{16}$.

Se definió "ingresado por varicela" a todo caso cuyo diagnóstico principal incluyera cualquier código de varicela. Para el cálculo de la tasa de ingresos por varicela se utilizaron las poblaciones estimadas por edad del INE. 
Se calculó la estancia media de los ingresos por varicela y $\mathrm{HZ}$ a partir de las fechas de ingreso y de alta del paciente.

Se definió "caso de varicela en ingresado por otra causa" aquél cuyo diagnóstico principal (motivo de ingreso) fue una causa diferente a varicela, pero presentaba algún código de enfermedad en algún otro campo diagnóstico.

Para valorar la influencia de la vacuna se representan los datos divididos en dos períodos, de 1997 a 2003 y de 2005 a 2007. Se ha excluido el año 2004 por ser un año con un incremento importante de casos al detectarse la mayor epidemia del período.

Se presentan los datos de hospitalización por varicela y de varicela en hospitalizados por otra causa, y de HZ por edad, sexo y las complicaciones.

Las defunciones por varicela y $\mathrm{HZ}$ se obtuvieron de la base de datos disponible en el Centro Nacional de Epidemiología, procedente del registro de mortalidad del Instituto Nacional de Estadística, seleccionando los códigos de causa de muerte de la CIE10 (B010.-B01. para varicela y B02.1
B02.9 para herpes zoster) desde 1999 hasta 2007, por grupos de edad y sexo.

\section{RESULTADOS}

Incidencia de varicela y HZ: Durante el periodo 1997-2007 se notificaron a la RENAVE una media anual de 197.137 casos de varicela (tasa media anual de 479,31 por 100.000 habitantes), con una tendencia decreciente y dos importantes ondas epidémicas, en 1999 y 2004. Los años 2005 y 2007 son los de menor incidencia del período (figura 1).

Durante el año 2004 la mayoría de las CCAA presentaron incrementos importantes en la incidencia de varicela contribuyendo a la mayor onda epidémica registrada en España desde 1997, si bien algunas CCAA, como Asturias, Canarias, Cantabria, Castilla la Mancha, Navarra y Melilla, experimentaron el aumento en los años posteriores.

Los años 2005 y 2007 son los de menor incidencia del período: el 2005, año de menor incidencia en España y en 5 CCAA (Canarias, Cataluña, Galicia, Murcia, La Rioja) y el 2007 año de menor incidencia del período en 6 CCAA: Baleares, Castilla

Figura 1

Incidencia de varicela por 100000 habitantes en España. RENAVE 1997-2007

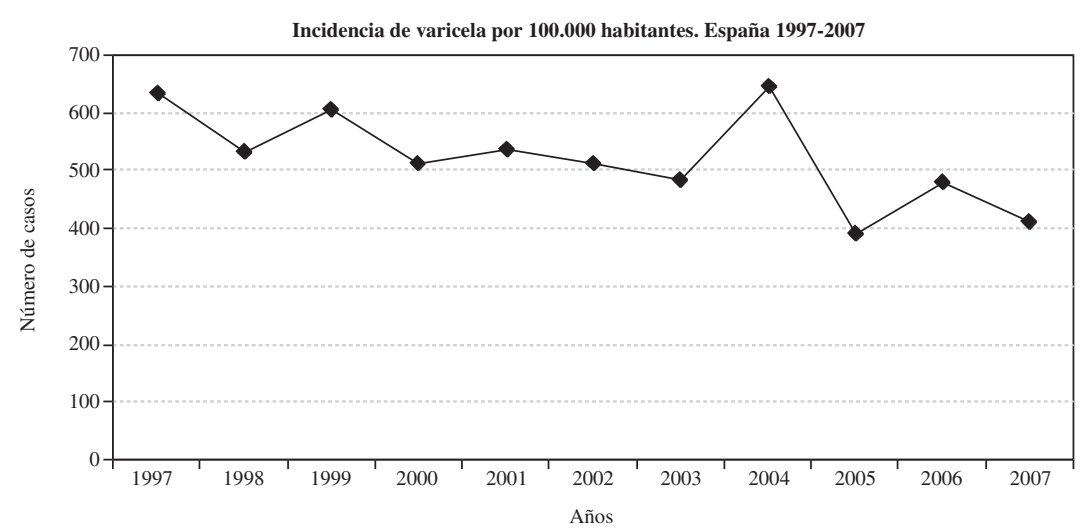

Fuente: RENAVE. 
la Mancha, Castilla y León, Comunidad Valenciana, Madrid y Navarra. Sin embargo durante 2007, 3 CCAA presentan tasas superiores a la media del período, Asturias, Cantabria y País Vasco, CCAA que presentaron la onda epidémica más tardíamente.

El análisis de los datos desagregados por edad del año 2007 muestra que el 88,1\% de los casos son menores de 15 años, con el 79,8 $\%$ entre las edades de 1 a 9 años ( 1 a 4 años: $50,6 \%$ y 5 a 9 años: $29,2 \%$ ) y el $4,5 \%$ menor de 1 año. El 9,9\% de los casos tienen entre 15 y 44 años, y el 1,1 \% son mayores de 44 años.

No se observan cambios en la distribución por edad, con relación a los datos disponibles para los años anteriores a 2007, a través de la vigilancia centinela en algunas CCAA (figura 2).

La distribución media por edad de los casos de HZ de las CCAA que realizan vigilancia epidemiológica, muestra un claro incremento con la edad, con el $87 \%$ de los casos en mayores de 25 años, y un 3,7\% en menores de 9 años(rango 82\%-89\%).

No se obtuvo información sobre el sexo ni el estado vacunal de los casos para el año 2007.
Morbilidad: Hospitalización y complicaciones. Durante 1997-2007 ingresó un promedio anual de 1.311 casos por varicela (3,1 por 100.000 habitantes y 6 por 1.000 casos notificados a la RENAVE). El año 2004 fue el año de mayor número de ingresos por varicela (4,2 por 100.000 habitantes), coincidiendo con el año de mayor incidencia. Se observó una tendencia creciente en la proporción de casos incidentes que eran ingresados, a excepción del año 2005, que podría deberse al incremento de cobertura del CMBD desde su inicio (figura 3).

El 59\% de las personas ingresadas fueron hombres, no siendo esta diferencia estadísticamente significativa, así como tampoco lo fue la distribución por edad, similar para ambos sexos, siendo el $62 \%$ de los ingresados en todo el período menor de 15 años.

La mayor incidencia de ingresos por varicela por 100.000 habitantes se produjo en los menores de 1 año, seguidos de los de 1- 4 años y fue superior en varones en todas las edades. No se observaron cambios significativos en la distribución por edad durante los ingresos en los períodos estudiados (1997-2003; 2005-2007) y tampoco en las complicaciones (figura 4).

Figura 2

Casos de varicela por grupos de edad. RENAVE 2007

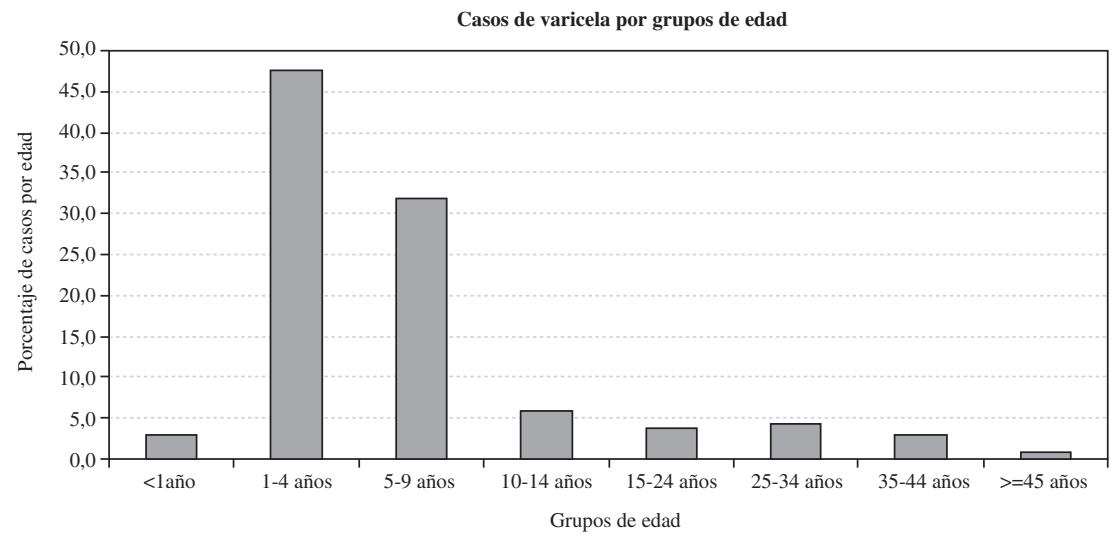

Fuente: RENAVE. 
Figura 3

Número de casos de ingresados por varicela y de casos de varicela ingresados por otras causas por año de ingreso. CMBD 1997-2007



El $49 \%$ de las personas ingresadas por varicela tuvo complicaciones, $50 \%$ mujeres y $51 \%$ hombres. La frecuencia de complicaciones varió con la edad: el $38 \%$ de los menores de 15 años ingresados por varicela presentó complicaciones, frente al $56 \%$ de los mayores de 14 años. El mayor porcentaje de complicaciones se observó

Tabla 1

Ingresados por varicela, varicelas ingresadas por otras causas y frecuencia de complicaciones por edad. CMBD 19972007

\begin{tabular}{|l|c|c|c|c|c|}
\hline \multicolumn{1}{|c|}{ Grupo de edad } & Ingresados por varicela & \% de complicaciones & Neumonitis & Encefalitis & Otras complicaciones \\
\hline$<1 \mathrm{a}$ & 1.816 & 23,5 & 5,8 & 0,3 & 15,0 \\
\hline $1-4 \mathrm{a}$ & 4.936 & 48,6 & 14,3 & 5,4 & 26,8 \\
\hline $5-9 \mathrm{a}$ & 1.552 & 44,6 & 10,2 & 11,3 & 21,5 \\
\hline $10-14 \mathrm{a}$ & 414 & 34,8 & 5,3 & 11,4 & 15,7 \\
\hline $15-24 \mathrm{a}$ & 600 & 47,8 & 29,2 & 5,8 & 13,3 \\
\hline $25-34 \mathrm{a}$ & 2.088 & 65,1 & 55,4 & 1,8 & 9,1 \\
\hline $35-44 \mathrm{a}$ & 1.678 & 68,6 & 60,3 & 1,7 & 9,1 \\
\hline$>=45$ & 1.020 & 44,1 & 26,7 & 4,7 & 14,5 \\
\hline Total & $\mathbf{1 4 . 1 0 4}$ & $\mathbf{4 9 , 0}$ & $\mathbf{2 5 , 6}$ & $\mathbf{4 , 6}$ & $\mathbf{1 8 , 2}$ \\
\hline \multicolumn{1}{|c|}{ Grupo de edad } & Varicelas ingresadas por otra causa & $\mathbf{6}$ de complicaciones & $\mathbf{N e u m o n i t i s}$ & $\mathbf{E n c e f a l i t i s}$ & Otras complicaciones \\
\hline & 941 & 10,8 & 4,7 & 0,4 & 6,1 \\
\hline $1-4 \mathrm{a}$ & 3.184 & 16,6 & 7,0 & 0,7 & 11,4 \\
\hline $5-9 \mathrm{a}$ & 909 & 14,4 & 7,9 & 0,9 & 10,0 \\
\hline $10-14 \mathrm{a}$ & 210 & 19,5 & 9,0 & 1,9 & 13,8 \\
\hline $15-24 \mathrm{a}$ & 495 & 10,7 & 5,9 & 2,0 & 5,7 \\
\hline $25-34 \mathrm{a}$ & 1.274 & 12,6 & 9,3 & 1,7 & 5,2 \\
\hline $35-44 \mathrm{a}$ & 565 & 23,7 & 15,8 & 2,5 & 11,0 \\
\hline$>=45$ & 581 & 23,9 & 12,0 & 2,6 & 13,9 \\
\hline Total & $\mathbf{8 . 1 5 9}$ & $\mathbf{1 5 , 8}$ & $\mathbf{8 , 9}$ & $\mathbf{1 , 6}$ & $\mathbf{9 , 6}$ \\
\hline
\end{tabular}


Figura 4

Porcentaje y tasa de ingreso por varicela por sexo y grupo de edad y porcentaje de complicaciones sobre los ingresos y cada una de ellas con respecto al total de casos complicados. CMBD comparación de los períodos 1997-2003 y 2005-2007



Complicaciones de la varicela en los ingresados por varicela entre 1997-2003



$\%$ Complicaciones



entre los adultos de 35 a 44 años (69\%) y 25 a 34 años $(65 \%)$. La complicación más frecuente fue la neumonitis, complicación que presentó el $26 \%$ de los ingresados por varicela, siendo del $43 \%$ en mayores de 14 años y del $9 \%$ en menores de esta edad. La encefalitis estuvo presente en el $4,6 \%$ de los ingresados y fue más frecuente en menores de 15 años $(7,1 \%)$ que en los mayores $(3,5 \%)$. "Otras complicaciones", 
Figura 4 (continuación)

Porcentaje y tasa de ingreso por varicela por sexo y grupo de edad y porcentaje de complicaciones sobre los ingresos y cada una de ellas con respecto al total de casos complicados. CMBD comparación de los períodos 1997-2003 y 2005-2007

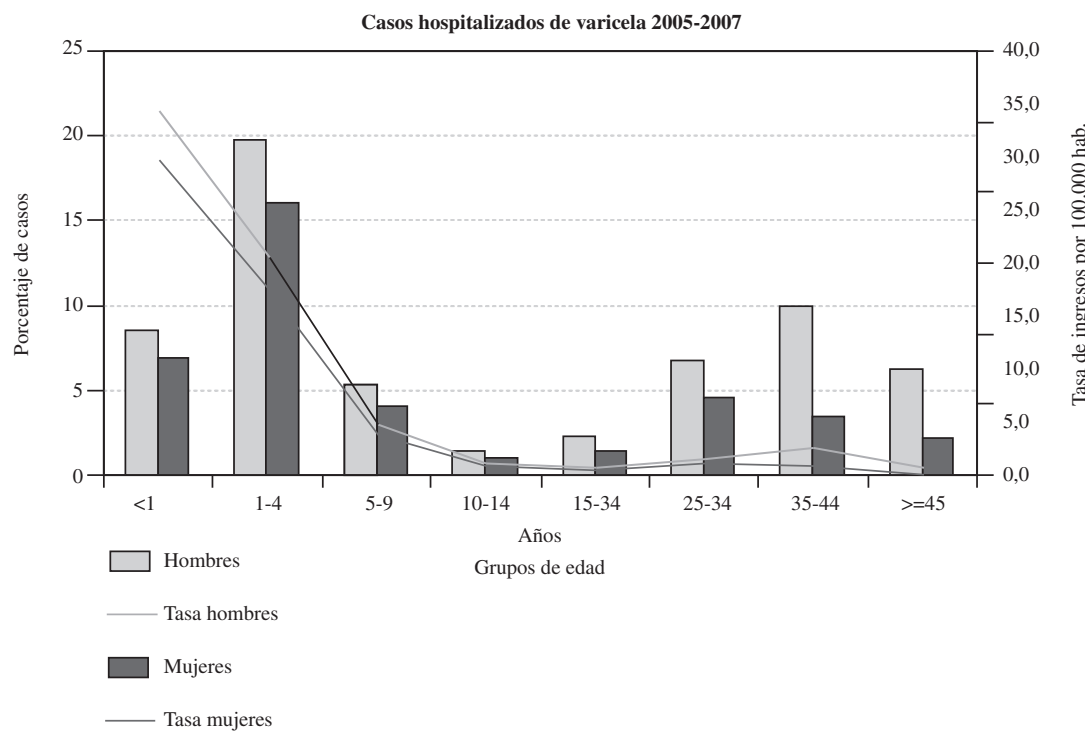

Complicaciones de la varicela en los ingresados por varicela entre 2005-2007

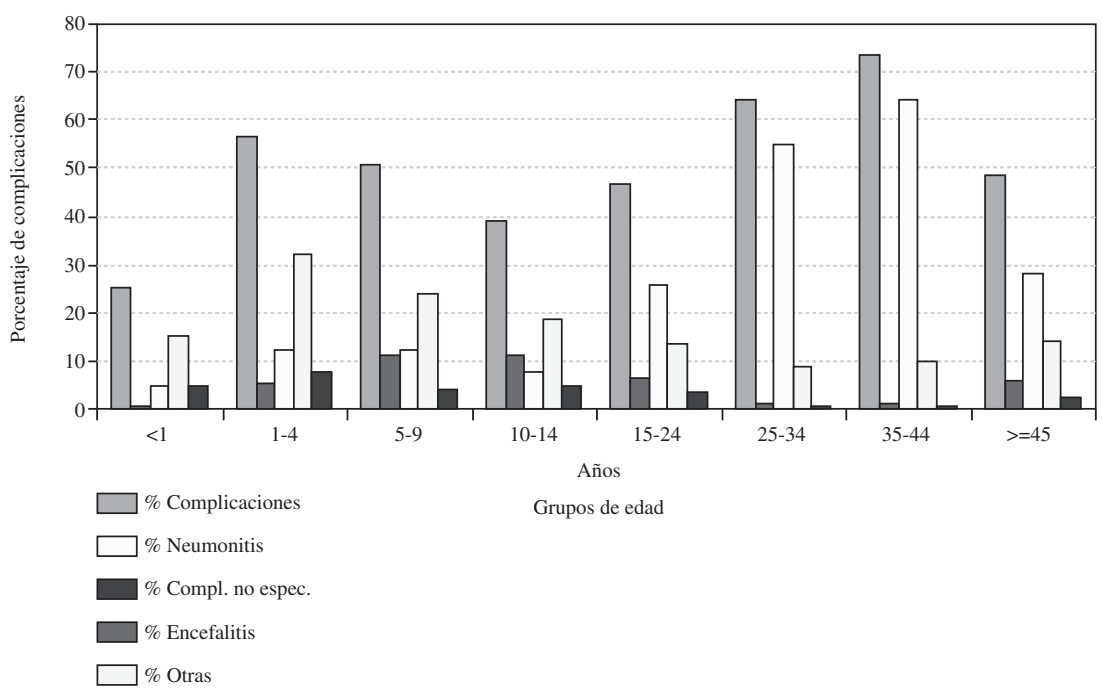

entre las que se encuentran las cutáneas las padecieron el $18,2 \%$ de los ingresados, y fueron más frecuentes en los menores de $15(20 \%)$ que en los mayores de esta edad $(11,5 \%)$ (tabla 1$)$.
El promedio anual de "varicela en ingresados por otras causas" fue de 742 casos $(15,5$ por 100.000 altas hospitalarias totales), con un importante incremento durante el año 2004 (23,4 por 100.000 altas hospi- 
talarias) que fue el de mayor incidencia de morbilidad por esta causa. Estos casos representaronn el 32-36\% constante durante el período de estudio entre todos los casos hospitalizados con varicela.

El 49\% de los casos de "varicela en ingresados por otras causas" fueron mujeres y el $50 \%$ niños de 0-4 años, disminuyendo la frecuencia de varicela entre ingresados por otras causas con la edad, con un incremento en las edades de 25 a 34 años a causa de un aumento de la frecuencia en mujeres. El 16\% tuvieron complicaciones, con una mayor frecuencia en hombres (9\% vs 6,7\%) en todos los grupos de edad. La complicación más frecuente fue "otra complicación sin especificar" $(9,5 \%)$ que incluyó las complicaciones cutáneas, siendo la más frecuente en todas las edades, excepto en los pacientes de 35-44 años, entre los que la complicación más frecuente fue la neumonitis (tabla 1).

En las personas ingresadas por varicela la estancia media fue de 6,5 días (DE: 6,9) y el $90 \%$ de los casos estuvieron ingresados menos de 11 días. Los casos de varicela ingresados por otras causas tuvieron una estancia media de 8,5 días (DE: 15,9) y el $90 \%$ de los casos estuvieron ingresados menos de 17 días.
Con respecto al HZ, durante 1997 a 2007 la tasa media anual de ingresos fue de 2,5 por 100.000 habitantes, y por edad de 2,7 ingresos por 100.000 habitantes mayores de 14 años y 1,03 por 100.000 habitantes menores de 15 años (tabla 2). La incidencia de ingresos por esta causa permaneció constante durante todo el período en ambos grupos de edad y fue similar para ambos sexos. La tasa media anual de casos de HZ entre personas hospitalizadas por otras causas fue de 44,03 por 100.000 ingresados; el $87 \%$ mayores de 45 años. Desde 1997 la frecuencia de casos entre hospitalizados por otras causas ha mantenido una tendencia ascendente, con los máximos en 2005-2007 (figura 5).

La estancia media en los ingresados por HZ es de 9,0 días con una desviación estándar de 8,7 mientras que los casos de $\mathrm{HZ}$ ingresados por otras causas permanecen más días hospitalizados $(14,5)$ con una desviación estándar de 18,0.

Los casos de varicela notificados agregados en períodos cuatrisemanales, tienen un patrón estacional que se mantiene a lo largo de toda la serie y de predominio a finales de primavera e inicios de verano, que se corresponde con el patrón epidemiológico típico de esta enfermedad en las áreas tem-

Tabla 2

Casos de Herpes zóster y tasas de ingresos por $\mathbf{1 0 0 . 0 0 0}$ habitantes. CMBD 1997-2007

\begin{tabular}{|l|c|c|c|c|c|c|}
\hline \multirow{2}{*}{} & \multicolumn{2}{|c|}{$<\mathbf{1 4}$ años } & \multicolumn{2}{c|}{$>\mathbf{1 4}$ años } & \multicolumn{2}{c|}{ Total } \\
\cline { 2 - 7 } & Casos & Tasa*100.000 & Casos & Tasa* 100.000 & Casos & Tasa* 100.000 \\
\hline 1997 & 52 & 0,85 & 832 & 2,51 & 884 & 2,25 \\
\hline 1998 & 67 & 1,11 & 930 & 2,75 & 997 & 2,50 \\
\hline 1999 & 52 & 0,87 & 873 & 2,55 & 925 & 2,30 \\
\hline 2000 & 73 & 1,24 & 981 & 2,83 & 1.054 & 2,60 \\
\hline 2001 & 64 & 1,09 & 1.023 & 2,90 & 1.087 & 2,64 \\
\hline 2002 & 65 & 1,10 & 1.014 & 2,82 & 1.079 & 2,58 \\
\hline 2003 & 62 & 1,03 & 982 & 2,68 & 1.044 & 2,44 \\
\hline 2004 & 50 & 0,82 & 1.027 & 2,77 & 1.077 & 2,49 \\
\hline 2005 & 64 & 1,02 & 979 & 2,59 & 1.043 & 2,36 \\
\hline 2006 & 77 & 1,21 & 955 & 2,49 & 1.032 & 2,31 \\
\hline 2007 & 69 & 1,06 & 1.003 & 2,66 & 1.072 & 2,42 \\
\hline Total & $\mathbf{6 9 5}$ & $\mathbf{1 , 0 4}$ & $\mathbf{1 0 . 5 9 9}$ & $\mathbf{2 , 6 9}$ & $\mathbf{1 1 . 2 9 4}$ & $\mathbf{2 , 4 5}$ \\
\hline
\end{tabular}


Figura 5

Número de ingresos por herpes zóster y herpes zóster en ingresados por otro motivo por sexo y año de ingreso. CMBD 1997-2007



pladas en cuanto a la varicela, no ocurre así con el herpes zoster como se ve en los ingresos por esta enfermedad. (figura 6).

Mortalidad. El número de muertes por varicela durante 1999 y 2007 osciló entre cuatro y catorce defunciones anuales con una tasa media anual de 0,02 defunciones por 100.000 habitantes y 4,8 muertes por 100.000 casos de varicela. El $80 \%$ de las defunciones registradas se han producido en personas mayores de 25 años, concentrándose en las edades de 25 a 44 años (44\% de las defunciones). Las mayores

Tabla 3

Mortalidad por grupos de edad. Varicela 1999-2007

\begin{tabular}{|c|c|c|c|c|c|c|c|c|c|c|c|c|c|c|c|c|c|c|c|c|c|c|}
\hline \multicolumn{23}{|c|}{ Defunciones por varicela (INE) 1997-2007 } \\
\hline \multirow{2}{*}{ Edad } & \multicolumn{2}{|c|}{1999} & \multicolumn{2}{|c|}{2000} & \multicolumn{2}{|c|}{2001} & \multicolumn{2}{|c|}{2002} & \multicolumn{2}{|c|}{2003} & \multicolumn{2}{|c|}{2004} & \multicolumn{2}{|c|}{2005} & \multicolumn{2}{|c|}{2006} & \multicolumn{2}{|c|}{2007} & \multicolumn{2}{|c|}{ Total } & \multirow{2}{*}{$\begin{array}{c}\text { Total } \\
\text { por edad }\end{array}$} & \multirow{2}{*}{$\%$ por edad } \\
\hline & H & $\mathbf{M}$ & $\mathbf{H}$ & $\mathbf{M}$ & $\mathbf{H}$ & $\mathbf{M}$ & $\mathbf{H}$ & $\mathbf{M}$ & $\mathbf{H}$ & $\mathbf{M}$ & $\mathbf{H}$ & $\mathbf{M}$ & $\mathbf{H}$ & $\mathbf{M}$ & $\mathbf{H}$ & $\mathbf{M}$ & H & $\mathbf{M}$ & H & $\mathbf{M}$ & & \\
\hline$<1$ año & & & & & & & & & & & & & & & & 1 & & & 0 & 1 & 1 & 1,19 \\
\hline 1-4 años & & 1 & 1 & & 1 & & 1 & 3 & & 2 & & & & & & & 1 & & 4 & 6 & 10 & 11,90 \\
\hline 5-9 años & 1 & 1 & & & & & & & & & & & & & & & & & 1 & 1 & 2 & 2,38 \\
\hline 10-14 años & & 1 & & & 1 & & & & & & & & & & & & & & 1 & 1 & 2 & 2,38 \\
\hline 15-24 años & 1 & & & & & & & & 1 & & & & & & & & & & 2 & 0 & 2 & 2,38 \\
\hline 25-34 años & 1 & & & & & & & 3 & & & 2 & 1 & & & & 1 & & & 3 & 5 & 8 & 9,52 \\
\hline 35-44 años & & & 6 & 1 & & 1 & & & 4 & 1 & 2 & 3 & 4 & 2 & 2 & 3 & & & 18 & 11 & 29 & 34,52 \\
\hline 45-54 años & & & 1 & & & & & & & & 1 & & & & & & & 1 & 2 & 1 & 3 & 3,57 \\
\hline 55-64 años & & & & & & & 1 & & & & 2 & & & & 1 & & 1 & & 5 & 0 & 5 & 5,95 \\
\hline 65-74 años & 2 & & & & & & 2 & & 1 & & & & 1 & & & & & & 6 & 0 & 6 & 7,14 \\
\hline$>=75$ años & & & & & 1 & & & & 1 & 1 & 2 & 1 & 3 & & 3 & 1 & & 3 & 10 & 6 & 16 & 19,05 \\
\hline Total por sexo & 5 & 3 & 8 & 1 & 3 & 1 & 4 & \begin{tabular}{|l|}
6 \\
\end{tabular} & 7 & \begin{tabular}{|l|} 
\\
\end{tabular} & 9 & 5 & 8 & 2 & 6 & 6 & 2 & 4 & 52 & 32 & 84 & 100 \\
\hline Total & & 3 & 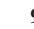 & 9 & 4 & 4 & 1 & 0 & 1 & 1 & 1 & 4 & 1 & 0 & 1 & 2 & s & 6 & & 34 & & \\
\hline
\end{tabular}


Tabla 4

Mortalidad por grupos de edad y sexo. Herpes zóster 1997-2007

\begin{tabular}{|c|c|c|c|c|c|c|c|c|c|c|c|c|}
\hline \multirow{3}{*}{ Años } & \multicolumn{12}{|c|}{ Grupos de edad y sexo } \\
\hline & \multicolumn{3}{|c|}{$<45$ años } & \multicolumn{3}{|c|}{ 45-49 años } & \multicolumn{3}{|c|}{ 60-69 años } & \multicolumn{3}{|c|}{$>70$ años } \\
\hline & $\mathbf{H}$ & M & Tasa $* 100.000$ & H M & Tasa & $* 100.000$ & H M & Tasa & $* 100.000$ & H M & Tasa & $* 100000$ \\
\hline 1999 & - & - & 0 & - & - & 0 & - & - & 0,00 & 3 & 11 & 0,31 \\
\hline 2000 & - & - & 0 & - & - & 0 & - & - & 0,00 & 3 & 9 & 0,26 \\
\hline 2001 & - & - & 0 & - & - & 0 & - & 1 & 0,03 & 8 & 9 & 0,35 \\
\hline 2002 & - & - & 0 & - & - & 0 & - & 1 & 0,03 & 3 & 9 & 0,24 \\
\hline 2003 & - & - & 0 & - & - & 0 & - & - & 0,00 & 5 & 10 & 0,29 \\
\hline 2004 & - & - & 0 & 1 & 1 & 0,03 & - & - & 0,00 & 3 & 11 & 0,27 \\
\hline 2005 & - & - & 0 & - & - & 0 & 1 & - & 0,03 & 5 & 9 & 0,26 \\
\hline 2006 & - & - & 0 & - & - & 0 & 3 & - & 0,07 & 10 & 10 & 0,36 \\
\hline 2007 & 1 & - & 0 & - & - & 0 & - & - & 0,00 & 6 & 12 & 0,32 \\
\hline Total & 1 & & 0,00 & 1 & 1 & $\mathbf{0 , 0 0}$ & 4 & 2 & 0,02 & 46 & 90 & 0,29 \\
\hline
\end{tabular}

Figura 6

Casos de varicela e ingresos por varicela y herpes zóster por cuatrisemana de diagnóstico. RENAVE, CMBD 1997-2006

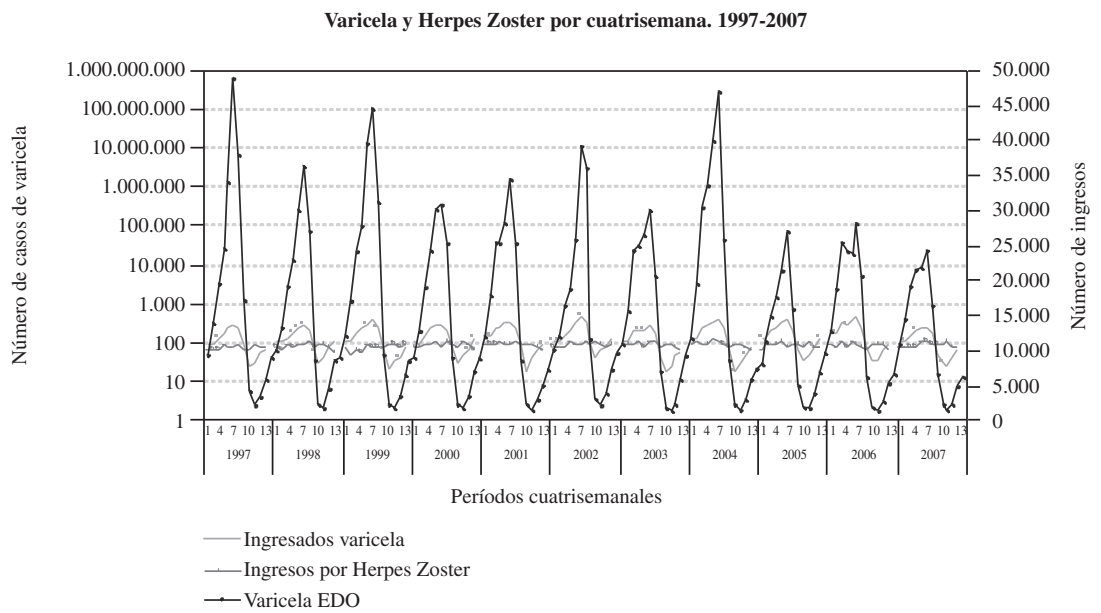

tasas de mortalidad se registran en los menores de 4 años, de 25 a 44 años y mayores de 65 años. Se observa un aumento en el número de defunciones a partir del año 2002, en el grupo de edad de 35 a 44 años y en mayores de 75 años, con un descenso importante en 2007 (tabla 3).

Durante el mismo período se han producido 145 muertes por $\mathrm{HZ}$. El número de defunciones anuales osciló entre 12 y 23 y una tasa media anual de 0,29 defunciones por 100.000 habitantes. El $95 \%$ de las defunciones se concentran en los mayores de 70 años y el $80 \%$ en mayores de 80 años. Entre los menores de 45 años hay 1 fallecido por HZ. El $36 \%$ de los fallecidos son hombres y el $64 \%$ son mujeres. (tabla 3 ).

\section{DISCUSIÓN}

El patrón de presentación de la varicela evidenciado en este estudio, es concordan- 
te con lo descrito en otros estudios previos $^{17-19}$ para países de nuestro entorno con vigilancia de esta enfermedad en ausencia de intervención; pues en muchos países miembros de la Unión Europea la varicela $\mathrm{y}$ el herpes zoster no son $\mathrm{EDO}^{20}$. Algunos países obtienen los datos a través de sistemas de vigilancia centinela basados en atención primaria.

La distribución por grupos de edad para el año 2007, no muestra diferencias significativas con respecto a la distribución en los años previos, ni antes ni tras la introducción de la vacuna, en aquellas CCAA que realizan la vigilancia centinela de esta enfermedad. Esta información está disponible tras el uso de la vacuna en los adolescentes seronegativos, lo cual no tendría porque ser diferente a la existente antes del uso de la vacuna, como se demuestra en aquellas CCAA que realizaban vigilancia centinela.

Se observa una disminución generalizada en prácticamente todas las CCAA durante los años 2005-2007 (período tras la incorporación de la vacunación), pero es difícil valorar el impacto de vacunación por la falta de cifras de cobertura vacunal y porque este período coincide con el período inmediato a la última epidemia cuyo máximo se registró en el año 2004 (año de máxima incidencia desde 1997) y por las limitaciones de la información disponible referente a la evolución de la incidencia y de la enfermedad.

Para verificar la disminución de la incidencia observada en los últimos años hará falta valorar la evolución en los próximos años, teniendo en cuenta el patrón epidémico diferencial y por edad, de cada CCAA y a nivel nacional.

La estacionalidad detectada en España corresponde con el patrón epidemiológico típico de áreas templadas, con un pico a finales de primavera e inicio de verano ${ }^{7,21}$.
En cuanto a los ingresos y las complicaciones por la enfermedad tampoco se observó un patrón claramente diferente por edad, ni en la hospitalización ni en las complicaciones, tras el año 2005 con la incorporación de la vacuna. Los mínimos cambios detectados hasta el momento al comparar el período 1997-2003 con 2005-2007, no son significativos y por ello habrá que evaluar los años siguientes en busca de cambios de patrón por edad o de afectación.

Un dato importante es que cerca de la mitad de los ingresados por varicela que es una enfermedad benigna, no tienen recogida ninguna complicación. Otro problema que se planteó es el análisis de aquellos casos que no tiene la varicela como diagnóstico principal sino concomitante. Se les ha denominado, varicelas ingresadas por otras causas, porque no parece muy probable un porcentaje tan elevado de contagio en los hospitales.

Las complicaciones descritas con más frecuentes, sobre-infecciones bacterianas en la infancia y neumonía en los adultos, coinciden con estudios tanto de ámbito nacional como internacional ${ }^{7,22,23}$.

Las complicaciones y la mortalidad son más frecuentes en mayores de 14 años, lo cual apoya la teoría de que en la infancia la varicela es una enfermedad benigna. La mortalidad en los años posteriores a la introducción de la vacuna tampoco ha manifestado un descenso hasta el año 2007. Habrá que valorar en años posteriores si este descenso se mantiene y puede estar jugando algún papel la vacuna, aún produciéndose en edades no vacunales.

El número de días de estancia hospitalaria es mayor en los casos de $\mathrm{HZ}$ que en los casos con varicela, al igual que en otros esudios $^{24,25}$. La tasa media anual de defunciones por herpes zoster es similar a la encontrada en otros estudios $(97 \%$ en $>60$ años $)^{23}$. 
Uno de los países con amplia experiencia en el uso de la vacuna son los Estados Unidos de América. Desde la introducción universal de la misma en 1995 se realizan estudios periódicos de la situación y cifran la disminución de la incidencia en un $90 \%$ y de la mortalidad en un $66 \%$, observándose sobre todo en el grupo objeto de vacunación de varicela (niños de 1-4 años) aunque no se registra descenso en personas mayores de 50 años $^{26}$.

Una de las limitaciones de este estudio es que la varicela es una enfermedad de declaración numérica y no tenemos información de casos por grupos de edad y sexo a nivel nacional. La información desagregada es muy reciente y por lo tanto poco útil para conocer el impacto del programa de vacunación.

Los indicadores propuestos para la vigilancia de la varicela en el año 2007, y la información correspondiente disponible en la actualidad, permiten caracterizar el patrón de presentación de la enfermedad en el período previo a la introducción de la vacuna en España. El escaso periodo de tiempo desde la introducción de la vacuna, junto con las limitaciones de la información disponible referente a la evolución de la incidencia por edad, hace difícil valorar el impacto de la vacunación en las edades dianas.

Para verificar la disminución de la incidencia observada en 2005-2007 hará falta valorar la evolución en los próximos años, teniendo en cuenta el patrón epidémico diferencial y por edad, de cada CCAA.

Para poder valorar adecuadamente el impacto de la vacunación es necesario disponer de un sistema que permita conocer las coberturas reales de vacunación por edad y CCAA, diferenciando las dosis administradas por el sector público y por el sector privado, para no infravalorar ni sesgar las estimaciones.

\section{AGRADECIMIENTOS}

A todos los responsables autonómicos por el envío de los datos.

\section{BIBLIOGRAFÍA}

1. Whitley RJ. Virus de la varicela zoster. En Enfermedades Infecciosas. Principios y práctica. $5^{\mathrm{a}}$ ed. Panamericana; 2000; 1931-8.

2. Gershon AA. Takahashi M and Seward J. Varicella Vaccine. In: Plotkin SA, Orenstein WA eds. Vaccines, 4th ed. Philadelphia: Saunders Company; 2004;783-823.

3. Lavanchy D. Varicela-Herpes Zoster. En: El control de las enfermedades transmisibles. American Public Health Association. Heymann LD editor. $18^{\mathrm{a}}$ ed. Publicación Científica y Técnica No .613 OPS. 2004;698-705.

4. Guess H, Broughton D, Melton L, Kurland LT. Population based studies of varicella complications. Pediatrics. 1986; 78(4 Pt 2): 723-7.

5. Hope-Simpson RE. The nature of herpes zoster: a long-term study and a new hypothesis. Proc R Soc Med. 1965;58:9-20.

6. Brisson M, Edmunds WJ, Law B, et al. Epidemiology of varicella zoster virus infection in Canada and the United Kingdom. Epidemiol Infect. 2001;127:305-314.

7. C. Amela Heras, I. Peña-Rey, I. Pachón del Amo, M.V. Martínez de Aragón. Actualización en varicela. Comentarios clínicos. Rev Clin Esp. 2006; 206(1): 48-9.

8. Clements D.A. Vacunación contra la varicela en la infancia. BioDrugs. 2000;14(1):49-61.

9. Committee of Infectious Diseases of the American Academy of Pediatrics. Recommendations for the use of live attenuated varicella vaccine. Pediatrics. 1995; 95: 791-796

10. Seward JF, Marin M, Vazquez M. Varicella vaccine effectiveness in the US vaccination program: a review. J Infect Dis. 2008; 197 Suppl 2:S82-S89.

11. Center for Disease Control and Prevention. Prevention of Varicella. Recommendations of the Advisory Committee on Immunization Practices (ACIP). MMWR, Morb Mortal Wkly Rep. 2007 June 22, /Vol.56/No.RR-4. 
12. Pachón I, Amela C, Martínez de Aragón M, Santaolalla P, Peña-Rey I, Cortés M. Varicela: Epidemiología y situación actual. Vacunas 2005. Disponible en: http://www.msc.es/ciudadanos/proteccionSalud/vacunaciones/docs/VARICELA1.pdf Acceso el 4 de agosto de 2009.

13. Calendarios de vacunación recomendados en España. Disponible en: http://www.msc.es/ciudadanos/proteccionSalud/infancia/vacunaciones/pro grama/vacunaciones.htm Acceso el 4 de agosto de 2009.

14. Estudio seroepidemiológico: situación de las enfermedades vacunables en España. Madrid: Instituto de Salud Carlos III; 1996. Disponible en: http://www.isciii.es/htdocs/centros/epidemiologia/pdf/SEROEPIDEMIOLOGICO.pdf. Citado el 4-08-2009.

15. Martínez de Aragón MV, Peña-Rey I, Suárez B. Propuesta para la vigilancia de la varicela y el herpes zoster.Disponible en: http://www.isciii.es/htdocs/ centros/epidemiologia/pdf/propuestavigilancia-varicela-2007.pdf. Citado el 4-08-2009.

16. Registro de altas de hospitalización-CMBD. SNS. Glosario de términos y definiciones. Ministerio de Sanidad y Política social. Febrero de 2008. Disponible en: http://pestadistico.msc.es/PEMSC25/Docs/ MANUAL\%20DE\%20DEFINICIONES\%20CMB D_Mar08.pdf. Acceso el 3 de agosto de 2009.

17. Martínez de Aragón MV, Peña-Rey I, Alcalde E. Informe sobre la situación de la varicela en España. Año 2007. Centro nacional de Epidemiología. Instituto de Salud Carlos III. Disponible en: http://www.isciii.es/htdocs/centros/epidemiologia/pdf/InformevaricelaCNE2007.pdf Acceso 3 de agosto de 2009 .

18. Socan M, Blasko M. Surveillance of varicella and herpes zoster in Slovenia, 1996-2005. Euro Surveill 2007; 12(2)[Epub ahead of print]. Available online: http://www.eurosurveillance.org/em/v12n02/1202227.asp
19. Choo P, Donahue J, Manson E, Platt R. The epidemiology of Varicella and its Complications. The Journal of Infectious Diseases 1995;172:706-712.

20. Bonanni P, Breuer J, Gershon A, Gershon M, Hryniewicz W, Papaevangelou V, Rentier B, Rümke H, Sadzot-Delvaux C, Senterre J, WeilOlivier C, Wutzler P. Varicella vaccination in Europe - taking the practical approach. BMC Med. 2009 May 28:7:26.

21. Varicella (chickenpox) trends. A Surveillance Community Network for Vaccine Preventable Infectious Diseases. Disponible en:http://www.euvac.net/graphics/euvac/trends_varicella.html

22. Séller T. Varicella-herpes zoster virus. En: Evans AS, editor. Viral infectious of humans. Epidemiology and control. Plenum Medical Book Company; 1989.

23. Pérez-Yarza E, Arranz L, Alustiza B, y cols, Grupo Varicela de Guipúzcoa. Hospitalizaciones por complicaciones de la varicela en niños menores de 15 años. An Pediatr. 2003; 59(3): 229-233.

24. Ziebold $\mathrm{CH}$, von Kries $\mathrm{R}$, Lang $\mathrm{R}$, Weigl J, Schmitt H. Severe complications of varicella in previously healthy children in Germany: A 1-year survey. Pediatrics. 2001;108: E79.

25. de Melker H, Berbers G, Hahne S, Rumke H, Hof $\mathrm{S}$ van den, de Wit A, Boot $\mathrm{H}$. The epidemiology of varicella and herpes zoster in the Netherlands: implications for varicella zoster virus vaccination. Vaccine. 2006; 24:3946-3952.

26. Gil A, San-Martín M, Carrasco P, González A. Epidemiology of severe varicella-zoster virus infection in Spain. Vaccine. 2004; 22:3947-3951.

27. Marin M, Meissner HC, Seward JF. Varicella prevention in the United States: a review of successes and challenges. Pediatrics. 2008; 122(3):e744e751. 